\title{
Educação estatística e parâmetros curriculares nacionais: algumas considerações
}

Danieli Walichinski

Guataçara dos Santos Junior

Eliana Claudia Mayumi Ishikawa

\begin{abstract}
Resumo
Este ensaio teórico tem como objetivo realizar uma discussão acerca das orientações dadas nos Parâmetros Curriculares Nacionais para os conteúdos de Estatística, Probabilidade e Combinatória, por entender que esse documento é a principal referência para o professor. A linha de pesquisa educacional que trata sobre esses conteúdos é a Educação Estatística. Para tal, realizou-se uma revisão bibliográfica, tendo como fonte de pesquisa os próprios Parâmetros Curriculares Nacionais, bem como, algumas das produções mais relevantes da área de Educação Estatística. Foram destacadas ao longo do texto algumas lacunas na proposta curricular brasileira, a começar pela falta de diferenciação entre a Educação Estatística e a Matemática. Entende-se que as discussões e considerações aqui apresentadas são oportunas tanto em cursos de formação inicial de professores, quanto em cursos de formação continuada, a fim de se efetivar a Estatística nas aulas de Matemática.
\end{abstract}

Palavras-chave: Parâmetros Curriculares Nacionais, Matemática, Educação Estatística.

\section{Abstract \\ Statistical education and parameters national curriculum: some considerations}

This theoretical essay aimshold a discussion about the Parameters National Curriculum given in guidelines for the contents of Statistics, Probability and Combinatorial, because we understand that this document is the main reference for the teacher. The line of educational research which deals with about of these contents is Statistical Education. To this end, a literature review was conducted, and as a source of research the Parameters National Curriculum themselves, as well as some of the most important productions of Statistical Education. Were highlighted throughout the text a few gaps in the brazilian curricular proposal, starting with the lack of differentiation between the Statistical Education and Mathematics.It is understood that discussions and considerations presented here are appropriate both in initial teacher training courses, and continuing education courses, in order to implement the Statistical Education in classes of Mathematics.

Keywords: parameters national curriculum, mathematics, statistical education. 


\section{Introdução}

Na década de 1970, um movimento em nível mundial pregou a necessidade de se romper com a cultura determinística, presente até então, nas aulas de Matemática. Esse movimento evidenciou a relevância da dimensão política e ética da utilização da Estatística na Educação Básica, bem como, a importância do desenvolvimento do raciocínio probabilístico (CAZORLA e UTSUMY, 2010). Para Cazorla, Kataoka e Silva (2010), tal movimento era a base do que atualmente denominase Educação Estatística (EE).

Acredita-se que esse movimento contribuiu para a inserção dos conteúdos de Estatística, Probabilidade e Combinatória nos currículos oficiais voltados para a Educação Básica de diversos países, como por exemplo, Itália e a França (1985), Estados Unidos da América (1988), Japão (1989), Espanha e Portugal (1991), dentre outros (LOPES, 1998). No Brasil, percebe-se que essa inclusão ocorreu tardiamente, pois os conteúdos de Estatística, Probabilidade e Combinatória só foram inseridos no currículo em 1997 com a publicação dos Parâmetros Curriculares Nacionais (PCN) para os anos iniciais do Ensino Fundamental, em 1998 com a publicação dos PCN para os anos finais do Ensino Fundamental e, em 1999 com a publicação dos PCN para o Ensino Médio.

Nos PCN referentes ao Ensino Fundamental à Estatística, à Probabilidade e à Combinatória fazem parte de um bloco de conteúdos denominado Tratamento da Informação. Por ser a primeira vez que esses conteúdos foram incluídos à proposta curricular brasileira, justificou-se a importância dos mesmos no tocante à formação de cidadãos críticos, uma vez que tais conteúdos estão diretamente ligados à leitura, interpretação e análise de informações veiculadas, bem como, à previsão de situações e à tomada de decisões.

Da mesma forma, na EE existe uma preocupação em relação à formação do cidadão em geral. A literatura é rica em trabalhos que abordam a necessidade de se oferecer uma formação estatística e probabilística significativa para que os alunos, enquanto cidadãos tenham condições de melhor atuar na sociedade. Podem-se citar como exemplos, trabalhos de vários autores de diferentes países que defendem tal necessidade: Lopes (1998, 2003, 2008, 2010a, 2010b, 2012), Batanero e Godino (2002), Gal (2002), Garfield (2002), Carvalho (2003, 2006, 2009), Pfannkuch (2008), Lopes e Coutinho (2009), Jacobini et al. (2010), Batanero, Arteaga e Contreras (2011), BenZvi (2011), Campos, Wodewotzki e Jacobini (2011), Oliveira (2013), Souza (2013), além de outros.

Considera-se que nos PCN, elaborados com a finalidade de se construir um referencial para orientar o trabalho do professor (BRASIL, 1998), deveriam ser encontradas orientações suficientes para que os professores possam oferecer uma formação estatística e probabilística significativa aos alunos, de modo a favorecer o desenvolvimento das competências estatísticas e probabilísticas. Nesse sentido, este ensaio teórico objetiva realizar uma discussão acerca das orientações dadas nos PCN para os conteúdos referentes ao bloco Tratamento da Informação, ou seja, para os conteúdos tema da EE. 


\section{Educação estatística}

A EE apresenta em suas linhas de pesquisa, investigações acerca do currículo, tanto da Educação Básica, quanto do Ensino Superior; formação inicial e contínua de professores; erros e dificuldades dos alunos e, inserção de novas tecnologias (LOPES, 2006). Lopes (2010a, p. 52) também destaca a importância da EE:

"A Educação Estatística não apenas auxilia a leitura e a interpretação de dados, mas fornece a habilidade para que uma pessoa possa analisar e relacionar criticamente os dados apresentados, questionando e até mesmo ponderando sua veracidade".

Os objetivos da EE, de acordo com Campos, Wodewotzki e Jacobini (2011, p. 12) são:

- "promover o entendimento e o avanço da EE e seus assuntos correlacionados;

- fornecer embasamento teórico às pesquisas em ensino da Estatística;

- melhorar a compreensão da dificuldades dos estudantes;

- estabelecer parâmetros para um ensino mais eficiente dessa disciplina;

- auxiliar o trabalho do professor na construção de suas aulas;

- sugerir metodologias de avaliação diferenciadas, centradas em METAS estabelecidas e em COMPETÊNCIAS a serem desenvolvidas;

- valorizar uma postura investigativa, reflexiva e crítica do aluno, em uma sociedade globalizada, marcada pelo acúmulo de informações e pela necessidade de tomada de decisões em situações de incerteza".

Existem diferenças importantes entre Estatística e Matemática, que precisam ser consideradas, uma vez que para a Matemática, o determinismo é o centro de todo o processo; enquanto que a Estatística apresenta como foco a variabilidade dos dados, em que a incerteza se faz presente (WALICHINSKI; SANTOS JUNIOR, 2013). Corroborando com esse entendimento, Estevan (2010) afirma que a diferença fundamental entre a Estatística e a Matemática consiste na onipresença da variabilidade. Lopes e Coutinho $(2009$, p. 67) por sua vez, reforçam que:

"A indeterminação ou a incerteza dos dados distingue uma investigação estatística de uma exploração matemática, que tem uma natureza mais precisa: os conceitos e os procedimentos matemáticos são usados como ferramentas para resolver os problemas estatísticos, mas estes não são limitados por eles; o fundamental nos problemas estatísticos é que, pela sua natureza, não têm uma solução única e não podem ser avaliados como totalmente errados nem certos, 
devendo ser avaliados em termos da qualidade do raciocínio, da adequação dos métodos utilizados à natureza dos dados existentes".

Além disso, aspectos tais como "[...] a escolha da forma de organização dos dados, a interpretação, a reflexão, a análise e a tomada de decisões [...]" (CAMPOS; WODEWOTZKI; JACOBINI, 2011, p. 13) dão a Estatística uma peculiaridade diferente à da Matemática. Tais diferenças, apesar de fundamentais para a realização de um trabalho significativo com os conteúdos tema da EE, não foram contempladas nos PCN. Isso pode acarretar num ensino em que sejam valorizados o uso de fórmulas e os cálculos com números expressivos, reforçando ao aluno a visão determinista de mundo, levando-o a acreditar que cada pergunta deve ter uma resposta única para qualquer situação.Para reverter essa problemática, acredita-seque seja necessária a realização de discussões com os professores em cursos de formação continuada sobre as diferenças entre a Estatística e a Matemática, visto que essas não são abordadas nos PCN.

Em síntese, considera-se que o objeto de estudo da EE é o processo de ensino e aprendizagem de questões referentes à Estatística, à Probabilidade e à Combinatória em todos os níveis de ensino, com a finalidade de propiciar uma aprendizagem mais significativa ao aluno, contribuindo para o desenvolvimento de competências tanto estatísticas, quanto probabilísticas. Na sequência serão apresentadas algumas considerações a respeito de tais competências.

\section{Pensamento estatístico}

O pensamento estatístico requer principalmente a formulação de hipóteses, interpretação e análise de resultados obtidos levando em consideração diferentes pontos de vista e, reformulação de questões com base nos resultados obtidos. Tais capacidades se diferenciam da exatidão e do determinismo exigidos na Matemática. Para atingir esse nível cognitivo os estudantes precisam abrir mão do determinismo (CAMPOS; WODEWOTZKI e JACOBINI, 2011) e, incorporar a ideia de aleatoriedade (COSTA, 2007). Além disso, o aluno precisa ser levado a perceber a existência da variabilidade dos dados e, como essa pode influenciar nos resultados. Entretanto, essas capacidades não se desenvolvem de um momento para outro. Os alunos precisam explorar situações diversas que contemplem o desenvolvimento do pensamento estatístico ao longo de sua escolarização. Da mesma forma, Batanero, Arteaga e Contreras (2011) destacam a necessidade de uma cultura do aleatório para a vivência na sociedade atual.

Vários pesquisadores, dentre eles Costa (2007) e Ruiz, Batanero e Arteaga (2011) defendem a idéia de se estreitar os laços com a Estatística Inferencial já no Ensino Fundamental. Considera-se que dessa forma os alunos tenham maiores condições de desenvolver o pensamento estatístico, pois se for trabalhado apenas com as noções de Estatística Descritiva, conforme explicitam os PCN, estará se desenvolvendo somente o raciocínio estatístico. Porém, não quer dizer que devam ser realizados processos complexos de inferência. Deve-se trabalhar de maneira informal, de modo a 
preparar os estudantes para posterior formalização.

Para colocar em prática esse pensamento junto aos alunos, Jacobini et al. (2010) sugerem que os alunos sejam levados a recolher os dados, a analisar e interpretar os mesmos, além de divulgar os resultados obtidos. Essas recomendações também estão presentes nos PCN. Contudo, tais situações por si só não garantem o desenvolvimento do pensamento estatístico. Outras questões tais como: a visualização do processo de maneira global; o entendimento do significado das variações; a exploração dos dados e, a geração de questões não previstas inicialmente (JACOBINI et al., 2010) devem ser exploradas juntamente com o trabalho de coleta e organização de dados. Da mesma forma, considera-se que o desenvolvimento do pensamento passa por situações que envolvem: elaboração de perguntas, escolha de variáveis, elaboração de hipóteses, percepção da necessidade de descrever populações, conscientização da necessidade dos dados, análise, organização e interpretação dos dados, transnumeração, escolha da melhor forma de representação de dados e, reformulação de questões.

\section{Raciocínio estatístico}

O raciocínio estatístico pode ser definido como sendo "a maneira com que as pessoas raciocinam com ideias estatísticas e como percebem a informação estatística" (GARFIELD, 2002 apud SILVA, 2007, p. 33). Visando complementar essa definição, encontrou-se em Mendonça e Lopes (2010) a afirmação de que o raciocínio estatístico se refere ao raciocínio aplicado para se trabalhar com as ferramentas e com os conceitos estatísticos.

Segundo Jacobini et al. (2010, p. 73) o raciocínio estatístico envolve questões, tais como, “[...] variabilidade, distribuição, chance, incerteza, aleatoriedade, probabilidade, amostragem, testes de hipóteses [...]". O raciocínio estatístico ainda envolve situações que dizem respeito à interpretação de resultados com base em dados reais e, ao entendimento e explicação de um processo estatístico (Campos et al. 2011).

Campos, Wodewotzki e Jacobini (2011) consideram que desenvolver o raciocínio estatístico não é uma simples tarefa. Segundo esses autores, existem tipos específicos de raciocínio desejáveis que os estudantes adquiram. São eles:

“a) raciocínio sobre dados: reconhecer e categorizar os dados e usar as formas adequadas de representação.

b) raciocínio sobre representação dos dados: entender como os gráficos podem ser modificados para representar melhor os dados.

c) raciocínio sobre medidas estatísticas: entender o que representa as medidas de tendência central e de espalhamento e qual medida é a mais adequada em cada caso. 
d) raciocínio sobre incerteza: usar adequadamente ideias de aleatoriedade $e$ chance para fazer julgamentos sobre eventos que envolvem incerteza. Entender que diferentes eventos podem demandar diferentes formas de cálculo de probabilidade.

e) raciocínio sobre amostragem: entender a relação entre a amostra e a população, o que pode ser inferido com base em uma amostra e desconfiar de inferências feitas a partir de pequenas amostras.

f) raciocínio sobre associação: entender como julgar e interpretar a relação entre duas variáveis. Entender que uma forte correlação entre duas variáveis não quer dizer que uma cause a outra" (GARFIELD, 2002 apud CAMPOS et al., 2011, $p$. 481-482).

Observa-se que para esses autores, questões referentes à Probabilidade também dizem respeito ao raciocínio estatístico. Da mesma forma, Lopes (2012, p. 167) destaca a intersecção entre os temas comuns à $\mathrm{EE}$, como se pode notar:

"O conceito-chave da ciência estatística é a variabilidade, que implica na capacidade de perceber a existência da variação. O raciocínio estatístico tem a variabilidade como o centro do processo de fazer relações sobre o problema investigado, de elaborar a construção e a análise dos dados. A variabilidade presente nos dados determina uma forma de pensar que exige uma combinação de ideias, o que nos remete a uma intersecção entre os raciocínios combinatório, probabilístico e estatístico".

Portanto, percebe-se a necessidade de se desenvolver os conteúdos de Estatística e Probabilidade de forma articulada, visando o desenvolvimento tanto de competências estatísticas quanto de competências probabilísticas (competências estocásticas ${ }^{1}$ ).

Considera-se que o raciocínio estatístico está diretamente ligado à Estatística Descritiva. Ou seja, o raciocínio estatístico envolve questões tais como: leitura e interpretação de dados, coleta de dados, construção de diferentes tipos de gráficos, construção de diferentes tipos de tabelas, cálculo e interpretação das medidas de tendência central e, cálculo e interpretação das medidas de dispersão.

\section{Pensamento probabilístico}

Conforme destacado por Lugli e Lopes (2010), o pensamento probabilístico ainda é pouco

\footnotetext{
${ }^{1}$ Estocástica é um termo bastante comum na área de EE para tratar da Estatística e da Probabilidade de forma articulada.
}

R. B. E. C. T., vol 7, núm. 3, set-dez.2014 ISSN - 1982-873X

DOI: Em andamento. 
explorado na Educação Básica. Em sua tese, Lopes (2003) considera que muitos estudantes saem da escola sem desenvolver o pensamento probabilístico.

O pensamento probabilístico, de acordo com Lopes (2010b) possibilita realizar uma análise quantitativa sobre as chances de um fenômeno ocorrer ou não. Ainda para Lopes e Ferreira (2004), esse pensamento consiste, fundamentalmente, na capacidade de utilizar os conceitos probabilísticos na solução de problemas, o que envolve desde estratégias de resolução, até uma análise de resultados.

Além disso, o uso da linguagem probabilística é fundamental para o desenvolvimento desse tipo de pensamento, conforme destacado por Marocci e Nacarato (2013, p. 121):

"Dessa forma, entendemos que o trabalho com tarefas que envolvam a linguagem probabilística é de fundamental importância para o desenvolvimento do pensamento probabilístico, pois a apropriação de um vocabulário adequado se constituirá em ferramenta para esse pensamento".

Na visão de Lopes (2006, p.79):

"O desenvolvimento do pensamento probabilístico requer o reconhecimento de situações de acaso na vida cotidiana e no conhecimento científico, bem como a formulação e comprovação de conjecturas sobre o comportamento de fenômenos aleatórios simples e a planificação e realização de experiências nas quais se estude o comportamento de fatos que abarquem o azar. A partir dessas considerações, pode-se organizar situações didáticas que envolvam a observação de experimentos, com seus respectivos registros e análises, possibilitando a integração entre a Probabilidade e a Estatística. Nessa conjunção é que se terá o desenvolvimento do raciocínio estocástico".

Nesse mesmo entendimento, Santos e Grando (2011) destacam que não é possível tratar do pensamento probabilístico isolado da Estatística. Assim, de acordo com Lopes (2003), para que ocorra o desenvolvimento do pensamento probabilístico, é imprescindível a concepção de azar e de aleatoriedade, pois a partir dessas concepções, poderão ser organizadas situações de ensino que explorem a observação de experimentos, seguidos de análise e registros, possibilitando dessa forma a articulação entre a Estatística e a Probabilidade.

Com base nas investigações acerca do pensamento probabilístico, Lopes (2003, p. 64) apresenta algumas considerações sobre a prática educativa dessa competência:

"Têm-se discutido sobre a necessidade de se realizar experimentos de simulação - nos quais apareçam seqüencias aleatórias -, de se dar importância à utilização de vocabulário adequado para descrever e quantificar situações relacionadas ao 
azar e de se confeccionar tabelas de freqüências e gráficos para representação do comportamento de fenômenos aleatórios".

Além disso, para desenvolver o pensamento probabilístico, Marocci e Nacarato (2013) entendem que é necessário trabalhar com os estudantes tanto as noções subjetivas de Probabilidade, quanto às de caráter formal. Entende-se que o pensamento probabilístico envolve fundamentalmente a percepção do acaso e da aleatoriedade, além das noções básicas de Probabilidade.

\section{Raciocínio probabilístico}

O raciocínio probabilístico, segundo Lopes (2010b) permite maior desenvoltura diante de situações que requerem tomadas de decisões. Ainda de acordo com Lopes (2012, p. 168), o raciocínio probabilístico está associado ao raciocínio combinatório, “[...], ou seja, após a enumeração das possibilidades, pode-se analisar a chance e fazer previsões". Lopes (2012) enfatiza ainda que o raciocínio probabilístico intersecciona-se com o raciocínio estatístico, uma vez, que esse é fundamental no momento de se analisar dados construídos com base em um problema.

Em Batanero e Godino (2002) encontram-se orientações sobre como ajudar no desenvolvimento do raciocínio probabilístico dos alunos:

- Proporcionar uma ampla variedade de experiências que permitam observar os fenômenos aleatórios e diferenciá-los dos deterministas;

- Estimular a expressão de predições sobre o comportamento desses fenômenos e os resultados, assim como sua probabilidade;

- Organizar a coleta de dados de experimentação de modo que os alunos tenham possibilidade de contrastar suas predições com os resultados produzidos e revisar suas crenças;

- Ressaltar o caráter imprevisível de cada resultado isolado, assim como a variabilidade das pequenas amostras, mediante a comparação de resultados de cada aluno ou por partes;

- Ajudar a apreciar o fenômeno da convergência, mediante acumulação de resultados de toda a turma, e comparar a confiabilidade de pequenas e grandes amostras.

Marocci (2011) ressalta a importância de que o aluno desenvolva o raciocínio probabilístico desde o início do Ensino Fundamental, para que assim, tenha maiores condições de desenvolver também o pensamento probabilístico.

Concorda-se com Lugli e Lopes (2010) quando afirmam que à medida que nota-se a diferenciação entre a Matemática e a Estatística percebe-se a importância de se abordar 
integradamente a Estatística e a Probabilidade, integrando as diferentes formas de raciocínio e pensamento, pois o desenvolvimento dessas competências é que possibilitarão a análise de dados e consequentemente a tomada de decisão.

\section{A Educação Estatística nos PCN}

Os PCN foram elaborados com a finalidade de se construir um referencial para orientar o trabalho do professor, de modo a indicar "[...] os objetivos em termos das capacidades a serem desenvolvidas em cada ciclo, assim como os conteúdos para desenvolvê-las" (BRASIL, 1998, p. 16). Nesse documento, os conteúdos foram divididos em quatro blocos: Números e Operações; Espaço e Forma; Grandezas e Medidas; e Tratamento da Informação.

O bloco Tratamento da Informação diz respeito aos conteúdos referentes à Estatística, Probabilidade e Combinatória, ou seja, aos conteúdos tema da EE. Por ser a primeira vez que esses conteúdos foram inseridos à proposta curricular brasileira, destacou-se a sua relevância para a vida em sociedade.

"A demanda social é que leva a destacar este tema como um bloco de conteúdo,
embora pudesse ser incorporado aos anteriores. A finalidade do destaque é
evidenciar sua importância, em função de seu uso atual na sociedade" (BRASIL,
1998, p. 52).

Apesar do destaque dado nos PCN quanto à relevância dos conteúdos referentes ao bloco Tratamento da Informação, observa-se que nem é feito referência ao termo EE. Considera-se que os PCN deveriam ter discutido as diferenças entre a Estatística e a Matemática, pois a falta dessa discussão faz com que muitas vezes, os conteúdos relacionados à Estatística sejam abordados com ênfase apenas no cálculo, conforme já ressaltado.

Observa-se ainda que embora os PCN justifiquem a necessidade do trabalho com a Estatística e a Probabilidade pelo fato de se "[...] possibilitar o desenvolvimento de formas particulares de pensamento e raciocínio para resolver determinadas situações-problema [...]" (BRASIL, 1998, p. 134), não é discutido o significado dessas formas particulares de pensamento e raciocínio, ficando assim uma lacuna nesse documento. A falta dessa discussão pode fazer com que as competências estatísticas e probabilísticas sejam confundidas com o raciocínio e o pensamento matemáticos.

Da mesma forma, Lopes (2011) considera que as discussões e reflexões sobre o ensino de Estatística e Probabilidade apresentadas nos PCN não são suficientes. Segundo essa autora:

"Pensamos que os Parâmetros deveriam ter posto em maior evidência as questões relativas ao ensino da Probabilidade e da Estatística, considerando que tais temas nunca foram antes abordados em propostas curriculares brasileiras,

52 DOI: Em andamento.

R. Bras. de Ensino de C\&T 
além de não terem feito parte da formação inicial do professor" (LOPES, 1998, p. 112).

Souza (2013, p. 50) também aponta fragilidades nos PCN:

"Nos objetivos gerais, os PCN de Matemática orientam que é importante que o professor promova atividades que possibilitem aos alunos desenvolver atitudes de organização, investigação e perseverança; além disso, consideram fundamental os alunos assumirem, diante de sua produção, uma postura que os direcione a justificar e validar suas respostas e observar que situações de erro são comuns e que, a partir deles, também se pode aprender.

Tais orientações seguem uma linha de raciocínio mais exploratória e inferencial informal. Porém, os objetivos específicos apresentados pelos $P C N$ referem-se aos conteúdos de forma muito vaga. Os PCN limitam-se a orientar a coleta de informações, a construção de gráficos e tabelas com base em textos jornalísticos e científicos. Essa abordagem se assemelha à representacionista, pois a grande maioria dos jornais traz as informações prontas, não permitindo ao aluno a participação no processo de investigação".

Assim como Souza (2013), entende-se que os objetivos específicos elencados nos PCN para os anos finais do Ensino Fundamental tratam os conteúdos do bloco Tratamento da Informação de forma bastante vaga, o que pode contribuir para que esses conteúdos sejam trabalhados de modo superficial, sem a devida preocupação com o desenvolvimento das competências estatísticas e probabilísticas.

De acordo com os PCN, os objetivos específicos para o terceiro ciclo $\left(6^{\circ}\right.$ e $7^{\circ}$ ano) referentes ao bloco Tratamento da Informação visam ao desenvolvimento:

- "Do raciocínio combinatório, estatístico e probabilístico, por meio da exploração de situações de aprendizagem que levem o aluno a:

- coletar, organizar e analisar informações, construir e interpretar tabelas e gráficos, formular argumentos convincentes, tendo por base a análise de dados organizados em representações matemáticas diversas;

- resolver situações-problema que envolvam o raciocínio combinatório e a determinação da probabilidade de sucesso de um determinado evento por meio de uma razão" (BRASIL, 1998, p. 65).

Para o quarto ciclo ( $8^{\circ}$ e $9^{\circ}$ ano), o bloco Tratamento da Informação tem como objetivos o desenvolvimento:

- "Do raciocínio estatístico e probabilístico, por meio da exploração de situações de aprendizagem que levem o aluno a:

R. B. E. C. T., vol 7, núm. 3, set-dez.2014 ISSN - 1982-873X

DOI: Em andamento. 
- Construir tabelas de freqüência e representar graficamente dados estatísticos, utilizando diferentes recursos, bem como, elaborar conclusões a partir da leitura, análise, interpretação de informações apresentadas em gráficos e tabelas;

- "Construir um espaço amostral de eventos equiprováveis, utilizando o princípio multiplicativo ou simulações, para estimar a probabilidade de sucesso de um dos eventos" (BRASIL, 1998, p. 82).

Observa-se ainda que o desenvolvimento dos pensamentos estatístico e probabilístico não se constituem como objetivos para o terceiro ciclo, nem para o quarto ciclo, apesar da justificativa dada nos PCN de que a Estatística e a Probabilidade possibilitam o desenvolvimento de formas particulares de pensamento. Com isso, cabe destacar a necessidade de uma (re) organização curricular para tratar especificamente dos objetivos do ensino da Estatística, da Probabilidade e da Combinatória nos anos finais do Ensino Fundamental.

Além disso, considera-se que os conteúdos propostos para os ciclos que correspondem aos anos finais do Ensino Fundamental são apresentados de forma fragmentada, como se pode observar no trecho referente aos conteúdos propostos para o terceiro ciclo:

"Quanto ao bloco Tratamento da Informação, se nos ciclos anteriores os alunos começaram a explorar idéias básicas de estatística - aprendendo a coletar e organizar dados em tabelas e gráficos, a estabelecer relações entre acontecimentos, a fazer algumas previsões, a observar a freqüência de ocorrência de um acontecimento - neste ciclo é importante fazer com que ampliem essas noções, aprendendo também a formular questões pertinentes para um conjunto de informações, a elaborar algumas conjecturas e comunicar informações de modo convincente, a interpretar diagramas e fluxogramas" (BRASIL, 1998, p. 69-70).

Considera-se que a apresentação dos conteúdos propostos para cada ciclo nos PCN poderia ser mais bem organizada, de modo a definir mais precisamente o conteúdo específico que deve ser trabalhado, uma vez que esse documento se constitui em um referencial para o professor.

Mesmo no tópico de conteúdos que se referem aos conceitos e procedimentos acredita-se que os conteúdos poderiam ser melhor destacados. A seguir, são elencados os tópicos de conteúdos relacionados ao trabalho com os conceitos e procedimentos sugeridos para o terceiro ciclo:

- "Coleta, organização de dados e utilização de recursos visuais adequados (fluxogramas, tabelas e gráficos) para sintetizá-los, comunicá-los e permitir a elaboração de conclusões. 
- Leitura e interpretação de dados expressos em tabelas e gráficos.

- Compreensão do significado da média aritmética como um indicador da tendência de uma pesquisa.

- Representação e contagem dos casos possíveis em situações combinatórias.

- Construção do espaço amostral e indicação da possibilidade de sucesso de um evento pelo uso de uma razão" (BRASIL, 1998, p. 74-75).

Da mesma forma, são apresentados os conteúdos relacionados ao trabalho com os conceitos e procedimentos sugeridos para o quarto ciclo:

- 'Leitura e interpretação de dados expressos em gráficos de colunas, de setores, histogramas e polígonos de freqüência.

- Organização de dados e construção de recursos visuais adequados, como gráficos (de colunas, de setores, histogramas e polígonos de freqüência) para apresentar globalmente os dados, destacar aspectos relevantes, sintetizar informações e permitir a elaboração de inferências.

- Compreensão de termos como freqüência, freqüencia relativa, amostra de uma população para interpretar informações de uma pesquisa.

- Distribuição das freqüências de uma variável de uma pesquisa em classes de modo que resuma os dados com um grau de precisão razoável.

- Obtenção das medidas de tendência central de uma pesquisa (média, moda e mediana), compreendendo seus significados para fazer inferências.

- Construção do espaço amostral, utilizando o princípio multiplicativo e a indicação da probabilidade de um evento por meio de uma razão.

- Elaboração de experimentos e simulações para estimar probabilidades e verificar probabilidades previstas".

Observa-se que os conceitos e procedimentos indicados para o quarto ciclo foram melhor explorados, uma vez, que incentivam o trabalho tanto com os conteúdos pertinentes à Estatística, quanto às noções básicas de Probabilidade, por meio de situações que demandam a compreensão de conceitos e a construção de significados, em oposição à definição de termos e a aplicação de fórmulas. No entanto, considera-se que esses conceitos e significados deveriam ser melhor explorados. Ao se trabalhar com conteúdos de Estatística e Probabilidade, pesquisadores como Lopes (2008), Marocci (2011), Borba et al. (2011), além de outros, observam que é necessário refletir sobre quais conceitos devem ser abordados, com o intuito de se possibilitar aos estudantes o desenvolvimento de uma visão estatística e probabilística significativa. 
De acordo com os PCN (BRASIL, 1998), a finalidade do ensino da Estatística para os anos finais do Ensino Fundamental é fazer com que os estudantes possam construir procedimentos para coletar e organizar dados, comunicando-os por meio de tabelas e gráficos; calcular a média, a moda e a mediana, com o objetivo de interpretar dados estatísticos. Considera-se que os PCN enfatizam demais a necessidade de leitura e interpretação de gráficos e tabelas, gerando um falso entendimento de que o ensino de Estatística se resume a ler e interpretar gráficos e tabelas. Com isso, deixam a desejar orientações de atividades que contemplem uma investigação estatística, tais como: formulação de questões, levantamento de hipóteses, escolha das variáveis, representatividade de uma amostra, confrontação da hipótese com os resultados alcançados, levantamento de novos questionamentos após a verificação dos resultados. Corroborando com esse entendimento, Oliveira (2013) destaca a necessidade do rompimento da idéia de que o ensino de Estatística está vinculado apenas à leitura de gráficos e tabelas.

Quanto à Probabilidade, a principal intenção consiste em que o aluno possa compreender que muitos dos acontecimentos do cotidiano são de natureza aleatória e, que se podem identificar possíveis resultados para esses acontecimentos e, até mesmo estimar o grau de possibilidade acerca do resultado dos mesmos (BRASIL, 1998). Os PCN também colocam a necessidade de se explorar as noções de acaso e incerteza, em que o aluno possa realizar experimentos e observar eventos. Considera-se que essas orientações não tem sido suficientes. Faltam exemplos práticos de situações que envolvam fenômenos aleatórios, de modo a auxiliar efetivamente o trabalho do professor.

Além disso Goulart (2007, p. 78) destaca que os PCN poderiam melhor explorar certos aspectos referentes à Probabilidade, como se observa no trecho seguinte:

\footnotetext{
"Entretanto, ao propor que o ensino de probabilidade no ensino fundamental se limite a definição clássica de probabilidade e a espaços amostrais equiprováveis, os documentos, além de não dar continuidade nas atividades baseadas na definição freqüentista, que já eram propostas no segundo ciclo, induzem o docente a trabalhar de uma forma que poderá ocasionar a criação de obstáculos epistemológicos nos alunos, obstáculos estes, que podem causar prejuízos à aprendizagem futura".
}

Acredita-se que um dos maiores obstáculos epistemológicos criados na disciplina de Matemática, seja a presença marcante da visão determinista de mundo. Por isso, destaca-se a importância do desenvolvimento das competências probabilísticas e estatísticas ao longo da escolarização, no sentindo de romper com esse paradigma. Outra questão observada é que os PCN tratam a Estatística e a Probabilidade de forma isolada, o que contribui para que geralmente a Probabilidade seja trabalhada apenas no $9^{\circ}$ ano do Ensino fundamental, quando a mesma deveria ser tratada de forma gradual juntamente com a Estatística desde os anos iniciais. 
O objetivo do ensino da Combinatória, para os PCN (BRASIL, 1998), é levar o aluno a trabalhar com situações que envolvem diferentes tipos de agrupamentos, de modo a desenvolver o raciocínio combinatório e a compreensão do princípio multiplicativo para sua aplicação no cálculo de probabilidades. Considera-se essa articulação entre a Combinatória e a Probabilidade como sendo um dos pontos positivos apresentados nos PCN. Entretanto, os PCN limitam-se a sugerir o trabalho com situações que envolvem diferentes tipos de agrupamentos, sem destacar quais são eles. Com isso, salvas exceções, no Ensino Fundamental é trabalhado apenas situações do tipo produto cartesiano, ficando de lado situações que envolvem combinações, arranjos e, permutações. Essas situações podem ser trabalhadas por meio de diferentes estratégias de resolução, tais como: listagens, desenhos, árvores de possibilidades, sem o uso de fórmulas.

De um modo geral, pode-se dizer que os PCN mostram-se muito abrangentes ao tratar sobre os conteúdos de Estatística, Probabilidade e Combinatória, ao invés de elencar cada conteúdo específico que deve ser trabalhado. Assim como Evangelista Sobrinho (2010) e Mendonça (2008) considera-se que os PCN deveriam mostrar ao professor, passo a passo, o que e como ele deve trabalhar. Não que deva ser uma "receita" a ser seguida, mas é necessário que seja definido tudo o que deve ser trabalhado e, não deixado apenas subentendido como fazem os PCN. Essa falta de clareza que os PCN trazem a respeito dos conteúdos do bloco Tratamento da Informação se reflete também nos livros didáticos, que por sua vez, simplificam os conteúdos específicos desse bloco de Conteúdos, favorecendo apenas a aplicação de exercícios de Matemática. Vale ressaltar que desse modo, os objetivos da EE e mesmo os dos PCN ficam comprometidos, pois acredita-se que o livro didático é o principal recurso utilizado pelos professores.

\section{Considerações finais}

Apesar dos conteúdos de Estatística, Probabilidade e Combinatória fazerem parte do currículo de Matemática, existem diferenças importantes a serem consideradas em relação à Estatística e a Matemática. Para o trabalho com a Matemática exige-se a exatidão e o determinismo, enquanto que para o trabalho com os conteúdos de Estatística é necessário considerar a variação dos dados. Considera-se que a falta dessa discussão nos PCN, faz com que muitas vezes, os conteúdos de Estatística sejam abordados com ênfase em cálculos e não em qualidade de pensamento e raciocínio.

Considera-se ainda que apesar de enfatizarem a relevância do trabalho com os conteúdos de Estatística, Probabilidade e Combinatória para a vida na sociedade atual, os PCN não explicitam que é por meio do desenvolvimento de competências estatísticas e probabilísticas que será possível a realização de uma análise de dados e a tomada de uma decisão mais acertada, deixando a impressão de que o trabalho com a Estatística serve apenas para aprender a ler gráficos e tabelas que aparecem nos mais variados meios de comunicação. 
Quanto a essa ênfase acerca da necessidade de leitura e interpretação de gráficos e tabelas, acredita-se que com isso, ficam a desejar orientações de atividades que contemplem uma investigação estatística, tais como: formulação de questões, levantamento de hipóteses, escolha das variáveis, representatividade de uma amostra, confrontação da hipótese com os resultados alcançados, levantamento de novos questionamentos após a verificação dos resultados.

Outra questão observada é que os objetivos específicos elencados nos PCN para os anos finais do Ensino Fundamental tratam os conteúdos do bloco Tratamento da Informação de forma bastante vaga, o que pode contribuir para que esses conteúdos sejam trabalhados de modo superficial. Além disso, considera-se que as orientações dadas nos PCN não tem sido suficientes. Faltam exemplos práticos de situações que envolvam os conteúdos, de modo a auxiliar efetivamente o trabalho do professor. De um modo geral, pode-se dizer que os PCN mostram-se muito abrangentes ao tratar dos conteúdos de Estatística, Probabilidade e Combinatória, ao invés de elencar cada conteúdo específico que deve ser trabalhado.

Observa-se também que contrário às tendências para a $\mathrm{EE}$, os PCN tratam a Estatística e a Probabilidade de forma isolada. A necessidade de uma articulação entre esses conteúdos é fundamental para a realização de um trabalho mais significativo com esses conteúdos. Um aspecto positivo observado nos $\mathrm{PCN}$ é o incentivo para se trabalhar tanto à Estatística, quanto à Probabilidade com situações que demandem a compreensão de conceitos e a construção de significados, em oposição à definição de termos e a aplicação de fórmulas. Considera-se, porém, que esses conceitos e significados a serem trabalhados deveriam ser melhor abordados nos PCN.

Cabe destacar ainda a necessidade de uma (re) organização curricular para tratar especificamente dos objetivos do ensino da Estatística, da Probabilidade e da Combinatória nos anos finais do Ensino Fundamental. Entende-se que as discussões e considerações aqui apresentadas são oportunas tanto em cursos de formação inicial de professores, quanto em cursos de formação continuada, a fim de se efetivar a Estatística nas aulas de Matemática.

\section{Referências}

BATANERO, C. GODINO, J. D. Estocástica y sua didáctica para maestros. Departamento de la didáctica de la matemática - Facultad de ciências de la educación. Universidad de Granada, Granada, 2002.

BATANERO, C. ; ARTEAGA, P. ; CONTRERAS, J. M. El currículo de estadística em la enseñanza obligatoria. Revista de Educação Matemática e Tecnológica Ibero-Americana,v. 2, n. 2, p. 1-20, 2011.

BEN-ZVI, D. Statistical reasoning learning environment. EM TEIA: Revista de Educação

Matemática e Tecnológica Iberoamericana, v. 2, n. 2, p. 1-13, 2011. 
BORBA, R. et al . Educação Estatística no Ensino Básico: Currículo, pesquisa e prática em sala de aula. EM TEIA: Revista de Educação Matemática e Tecnológica Iberoamericana, v. 2, p. 1-18, 2011.

BRASIL. Ministério da Educação. Secretaria de Ensino Fundamental. Parâmetros curricularesnacionais: ensino fundamental ( $5^{\underline{a}}$ a $8^{a}$ série)/matemática. Brasília (DF): MEC/SEF, 1998.

CAMPOS, C. R. et al . Educação Estatística no contexto da Educação Crítica. Bolema. Boletim de Educação Matemática (UNESP. Rio Claro. Impresso), v. 24, p. 473-494, 2011.

CAMPOS, C.R ; WODEWOTZKI, M. L. L.; JACOBINI, O. Educação estatística: teoria e prática em ambientes de modelagem matemática. Belo Horizonte: Autêntica, 2011.

CARVALHO, C. Literacia estatística. In: I SEMINÁRIO DE ENSINO DE MATEMÁTICA, 1., 2003, Campinas. Anais... Campinas: COLE, 2003.

CARVALHO, C. Desafios à educação estatística. Boletim SPE. Lisboa (Portugal), p. 7-10, 2006. CARVALHO, C. Reflexões em torno do ensino e da aprendizagem da estatística: o caso dos gráficos. In: FERNANDES, José A.; et al.(Orgs) Actas do II encontro de probabilidades e estatística na escola. Braga (Portugal), p. 22-36, jan. 2009.

CAZORLA, I. ; KATAOKA, V. Y. ; SILVA, Cláudia B. da . Trajetórias e perspectivas da educação estatística no Brasil: um olhar a partir do GT 12. In: LOPES, C. E. ; COUTINHO, C. de Q. e S. ; ALMOULOUD, S. A. (Orgs.) Estudos e reflexões em educação estatística. Campinas (SP): Mercado de letras, 2010.

CAZORLA, I. ; UTSUMI, M. C. Reflexões sobre o ensino da estatística na educação básica. In: CAZORLA, I.; SANTANA, E. (Org.) Do tratamento da informação ao letramento estatístico. Itabuna (BA): Via Litterarum, 2010.

COSTA, A. A Educação Estatística na Formação do professor de Matemática. Dissertação (Mestrado em Educação), 2007, 153p. Programa de Pós-Graduação em Educação. Universidade São Francisco, Itatiba (SP), 2007.

ESTEVAN, E. J. G. (Res)significandoa educação estatística no ensino fundamental: análise de uma sequência didática apoiada nas tecnologias de informação e comunicação. 2010. 211f. Dissertação (Mestrado em Educação) - Universidade Estadual Paulista-Faculdade de Ciência e Tecnologia, Presidente Prudente (SP), 2010.

EVANGELISTA SOBRINHO, F. O raciocínio combinatório e probabilístico de alunos do 60 ano do ensino fundamental. 2010. 135f. Dissertação (Mestrado emEnsino de Ciências e Matemática). Universidade Cruzeiro do Sul, São Paulo (SP), 2010.

GAL, I. Adult's statistical literacy : meanings, components, responsabilities. International

DOI: Em andamento. 
Statistical Review, v. 70, n. 1, p. 1-25, 2002.

GARFIELD, J. The challenge developing statistical reasoning. Journal of Statisticsl Education, v. 10, n. 3, p. 1-11, 2002.

GOULART, A. O discurso sobre os conceitos probabilísticos para a escola básica. 2007. $88 \mathrm{f}$. Dissertação (Mestrado em Educação Matemática)-Pontifícia Universidade Católica de São Paulo, São Paulo (SP), 2007.

JACOBINI, O. et al. Temas contemporâneos nas aulas de estatística: um caminho para combinar aprendizagem e reflexões políticas. In: LOPES, C. E. ; COUTINHO, C. de Q. e S. ; ALMOULOUD, S. A. (Orgs.) Estudos e reflexões em educação estatística. Campinas (SP): Mercado de Letras, 2010.

LOPES, C. A. E. A probabilidade e a estatística no ensino fundamental: umaanálise curricular. 1998. 139 f. Dissertação (Mestrado em Educação) - Faculdade de Educação, Universidade Estadual de Campinas, Campinas (SP), 1998.

LOPES, C. A. E. O conhecimento profissional dos professores e suas relações com estatística e probabilidade na educação infantil. 2003. $281 \mathrm{f}$. Tese (Doutorado em Educação) - Faculdade de Educação, Universidade Estadual de Campinas, Campinas (SP), 2003

LOPES, C. A. E. Educação Matemática e Educação Estatística: intersecções na produção científica. In: ARAÚJO JR., C.F; AMARAL, L. H.. (Org.). Ensino de Ciências e Matemática: Tópicos em Ensino e Pesquisa. São Paulo: ANDROSS, 2006, v. , p. 177-196.

LOPES, C. E. O ensino da estatística e da probabilidade na educação básica e a formação dos professores. Cad. Cedes, Campinas (SP), v. 28, n. 74, p. 57-73, 2008.

LOPES, C. E. Os desafios para educação estatística no currículo de matemática. In: LOPES, Celi E. ; COUTINHO, Cileda de Q. e S. ; ALMOULOUD, Saddo A. (Orgs.) Estudos e reflexões em educação estatística. Campinas (SP): Mercado de letras, 2010a.

LOPES, C. E. A educação estatística no currículo de matemática: um ensaio teórico. IN: REUNIÃO ANUAL DA ANPED. 33., 2010b, Caxambu (MG). Anais...

LOPES, C. E. . A Educação Estocástica na Infância. Revista Eletrônica de Educação (São Carlos), v. 6, p. 160-174, 2012.

LOPES, C. E.; COUTINHO, C. Q. S. Leitura e escrita em Educação Estatística. In: LOPES, C. E.; NACARATO, A. M. Educação matemática, leitura e escrita: armadilhas, utopias e realidades. Campinas, SP: Mercado das Letras, 2009. p. 61-78.

LOPES, C. E.; FERREIRA, A. C. A estatística e a probabilidade no currículo de matemática da escola básica. IN: VII ENCONTRO NACIONAL DE EDUCAÇÃO MATEMÁTICA. 2004, Recife (PE). Anais...

LUGLI, L. C. ; LOPES, C. E. Avaliações externas e o Currículo do Ensino Médio: uma análise sobre as questões que envolvem Probabilidade. IN: II Enrede - UFSCAR. 2010, São Carlos (SP). Anais... 
MAROCCI, L. M. O movimento das significações probabilísticas proporcionado pela resolução de problemas e pela prática colaborativa numa turma de 10 ano do ensino médio. 2011. $233 \mathrm{f}$. Dissertação (Mestrado em Educação)-Universidade São Francisco, Itatiba (MS), 2011.

MAROCCI, L.M. ; NACARATO, A. M. . Um ambiente de aprendizagem baseado na resolução de problemas: a possibilidade de circulação de significações sobre probabilidade por meio da linguagem. Educação Matemática Pesquisa (Online), v. 15, p. 101-123, 2013.

MENDONÇA, L. O. A Educação estatística em um ambiente de modelagem matemática no ensino médio. 2008. 245f. Dissertação (Mestrado em Ensino de Ciências e Matemática).Universidade Cruzeiro do Sul, São Paulo (SP), 2008.

MENDONÇA, L. O. ; LOPES, C. E. O trabalho com educação estatística no ensino médio em um ambiente de modelagem matemática. In: LOPES, C. E. ; COUTINHO, C. de Q. e S. ; ALMOULOUD, S. A. (Orgs.) Estudos e reflexões em educação estatística. Campinas (SP): Mercado de letras, 2010. OLIVEIRA, D. As aprendizagens dos professores que ensinam Matemática para crianças ao se inserirem em um espaço formativo sobre Estocástica. 2013. 139f. Tese (Doutorado em Ensino de Ciências e Matemática)-Universidade Cruzeiro do Sul, São Paulo (SP), 2013.

PFANNKUCH, M. Training teachers to develop statistical thinking. In: BATANERO, C. et al. (Ed.). Teaching statistics in school mathematics. challenges for teaching and teacher education. Proceedings of the Joint ICMI Study 18 and 2008 IASE Round Table Conference. Monterrey, México, 2008

RUIZ,B.. ; BATANERO, C. ; ARTEAGA, P. Vinculación de la variable aleatória y estadística em la realización de inferências informales por parte de futuros professores. Bolema. Boletim de Educação Matemática (UNESP. Rio Claro. Impresso), v. 24, n. 39, p. 431-451, 2011.

SANTOS, J. A. F. L; GRANDO, R. C. O movimento das ideias probabilísticas no ensino fundamental: análise de um caso. Bolema. Boletim de Educação Matemática (UNESP. Rio Claro. Impresso), v. 24, n. 39, p. 561-584, 2011.

SILVA, C. B. Pensamento estatístico e raciocínio sobre variação: um estudo com professores de matemática. 2007. 354f. Tese (Doutorado em Educação) -Pontifícia Universidade Católica, São Paulo (SP), 2007.

SOUZA, L. O. O desenvolvimento profissional de professores em estatística: um projeto multidimensional de formação colaborativa. 2013. 195f. Tese (Doutorado em Ensino de Ciências e Matemática)-Universidade Cruzeiro do Sul, São Paulo (SP), 2013.

WALICHINSKI, D.; SANTOS JUNIOR, G. Educação estatística: objetivos, perspectivas e dificuldades. Revista Imagens da Educação, v. 3, n. 3, p. 31-37, 2013. 
DANIELI WALICHINSKI - Mestre em Ensino de Ciência e Tecnologia pela Universidade Tecnológica Federal do Paraná (UTFPR). Professora de Matemática da Secretaria da Educação do Estado do Paraná, Ponta Grossa-PR. E-mail: dani.walichinski@gmail.com GUATAÇARA DOS SANTOS JUNIOR -- Doutor em Ciências Geodésicas pela Universidade Federal do Paraná (UFPR). Professor do Programa de Pós-Graduação em Ensino de Ciência e Tecnologia (PPGCET), da Universidade Tecnológica Federal do Paraná (UTFPR) - Câmpus Ponta Grossa.E-mail: guata@utfpr.edu.br

ELIANA C. M. ISHIKAWA - Mestre em Engenharia de Produção pela Universidade Federal de Santa Catarina (UFSC). Aluna do curso de doutorado do Programa de Pós-Graduação em Ensino de Ciência e Tecnologia (PPGCET) da Universidade Tecnológica Federal do Paraná (UTFPR). Professora de Ciências da Computação da UTFPR - Câmpus Ponta Grossa. E-mail: eishikawa@utfpr.edu.br 